\title{
Article \\ CRISPR/Cas12-Based Ultra-Sensitive and Specific Point-of-Care Detection of HBV
}

\author{
Ronghua Ding ${ }^{1}$, Jinzhao Long ${ }^{1}$, Mingzhu Yuan ${ }^{1}$, Xue Zheng ${ }^{1}$, Yue Shen ${ }^{1}$, Yuefei Jin ${ }^{1} \mathbb{D}$, Haiyan Yang ${ }^{1}, \mathrm{Hao} \mathrm{Li}^{2}$, \\ Shuaiyin Chen $1, * \mathbb{D}$ and Guangcai Duan $1,3, *$ \\ 1 College of Public Health, Zhengzhou University, Zhengzhou 450000, China; rhd23@foxmail.com (R.D.); \\ LJZzzu@yeah.net (J.L.); 15660129935@163.com (M.Y.); zhengxue0313@163.com (X.Z.); \\ shenyue5151@163.com (Y.S.); jyf201907@zzu.edu.cn (Y.J.); yhy@zzu.edu.cn (H.Y.) \\ 2 State Key Laboratory of Pathogen and Biosecurity, Beijing Institute of Microbiology and Epidemiology, \\ Beijing 100071, China; lihao88663239@126.com \\ 3 Key Laboratory of Molecular Medicine in Henan Province, Zhengzhou 450000, China \\ * Correspondence: sychen@zzu.edu.cn (S.C.); gcduan@zzu.edu.cn (G.D.); \\ Tel.: +86-0371-6778-1405 (S.C. \& G.D.)
}

Citation: Ding, R.; Long, J.; Yuan, M.; Zheng, X.; Shen, Y.; Jin, Y.; Yang, H.; Li, H.; Chen, S.; Duan, G. CRISPR/ Cas12-Based Ultra-Sensitive and Specific Point-of-Care Detection of HBV. Int. J. Mol. Sci. 2021, 22, 4842. https://doi.org/10.3390/ ijms22094842

Academic Editor: Damjan Glavač

Received: 31 March 2021

Accepted: 29 April 2021

Published: 3 May 2021

Publisher's Note: MDPI stays neutral with regard to jurisdictional claims in published maps and institutional affiliations.

Copyright: (c) 2021 by the authors. Licensee MDPI, Basel, Switzerland. This article is an open access article distributed under the terms and conditions of the Creative Commons Attribution (CC BY) license (https:// creativecommons.org/licenses/by/ $4.0 /)$.

\begin{abstract}
Hepatitis B remains a major global public health challenge, with particularly high prevalence in medically disadvantaged western Pacific and African regions. Although clinically available technologies for the qPCR detection of HBV are well established, research on point-of-care testing has not progressed substantially. The development of a rapid, accurate point-of-care test is essential for the prevention and control of hepatitis B in medically disadvantaged rural areas. The development of the CRISPR/Cas system in nucleic acid detection has allowed for pathogen point-of-care detection. Here, we developed a rapid and accurate point-of-care assay for HBV based on LAMP-Cas12a. It innovatively solves the problem of point-of-care testing in $10 \mathrm{~min}$, particularly the problem of sample nucleic acid extraction. Based on LAMP-Cas12a, visualization of the assay results is presented by both a fluorescent readout and by lateral flow test strips. The lateral flow test strip technology can achieve results visible to the naked eye, while fluorescence readout can achieve real-time high-sensitivity detection. The fluorescent readout-based Cas12a assay can achieve HBV detection with a limit of detection of 1 copy $/ \mu \mathrm{L}$ within $13 \mathrm{~min}$, while the lateral flow test strip technique only takes $20 \mathrm{~min}$. In the evaluation of 73 clinical samples, the sensitivity and specificity of both the fluorescence readout and lateral flow test strip method were $100 \%$, and the results of the assay were fully comparable to qPCR. The LAMP-Cas12a-based HBV assay relies on minimal equipment to provide rapid, accurate test results and low costs, providing significant practical value for point-of-care HBV detection.
\end{abstract}

Keywords: Hepatitis B virus (HBV); CRISPR/Cas12a; LAMP; point-of-care detection

\section{Introduction}

Hepatitis B is an infectious disease caused mainly by liver lesions resulting from hepatitis B virus (HBV) infection, and is recognized as a global public health problem [1,2]. In 2015, the World Health Organization estimated that 257 million people worldwide have been infected with the HBV (HBsAg positive) and 887,000 died from chronic hepatitis B [3,4]. Approximately $68 \%$ of these infections were mainly recorded in developing countries in the medically poor western Pacific region and Africa [5,6]. The limited medical facilities contribute to the extremely low HBV detection rate in these areas. Therefore, HBV detection requires not only high sensitivity, but also point-of-care detection. At present, the clinical detection of HBV is mainly based on quantitative real-time PCR (qPCR) and serological testing. Although qPCR is highly sensitive, it requires specialized equipment and personnel, and is time-consuming, thus limiting its use in point-of-care testing [7,8]. HBV serological testing has been widely used in community hospitals due to its simplicity and rapid detection. However, its sensitivity, cross-reactivity, and specificity are poor. Additionally, it 
is less effective than nucleic acid testing, because the body does not produce the appropriate antigenic antibodies during the window period of infection [9-11]. Therefore, a rapid, accurate, low-cost, and convenient point-of-care testing technology for HBV testing should be developed.

In recent years, clustered regularly interspaced short palindromic repeats (CRISPR)associated (Cas), nuclease-based methods have provided a promising approach for rapidly adaptable, deployable detection [12,13]. Compared with other point-of-care assays, such as recombinant polymerase isothermal amplification (RPA) and loop-mediated isothermal amplification (LAMP), the CRISPR/Cas assay is more sensitive and specific [14]. Since the discovery of CRISPR/Cas detection technology, scientists have developed various detection platforms based on CRISPR/Cas systems, such as SHERLOCK (Cas13a), DETECTR (Cas12a), CDetection (Cas12b), and Cas14-DETECTR, which have successfully performed rapid, highly sensitive and accurate detection of various pathogens [15-18].

Here, we used LAMP amplification to address the limitations of poor amplification by RPA, the long PCR amplification time, and the need for specialized equipment for CRISPR/Cas detection [19]. Moreover, we addressed the challenge of sample nucleic acid extraction within $10 \mathrm{~min}$ by using rapid sample preprocessing. HBV DNA templates extracted from the sample preprocessing were amplified by LAMP, followed by mixing with the Cas12a-crRNA complex for detecting specific DNA target sequences. The presence of HBV DNA activates the collateral effect of Cas12a, then cleaves the fluorescent reporter release fluorescent signal. In addition, the combination of lateral flow test strip technology enables point-of-care test results to be visualized by the naked eye (Figure 1). This study is based on the LAMP method combined with the CRISPR/Cas12a detection system, which enables the detection of 1 copy/ $\mu \mathrm{L}$ HBV DNA in a short time without relying on specialized equipment. The method of point-of-care testing for HBV provided in this study has important implications for the diagnosis and clinical management of hepatitis B in developing countries, where medical equipment is lacking.
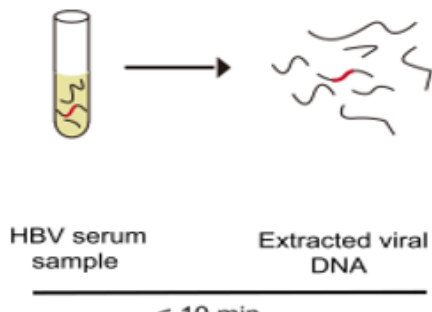
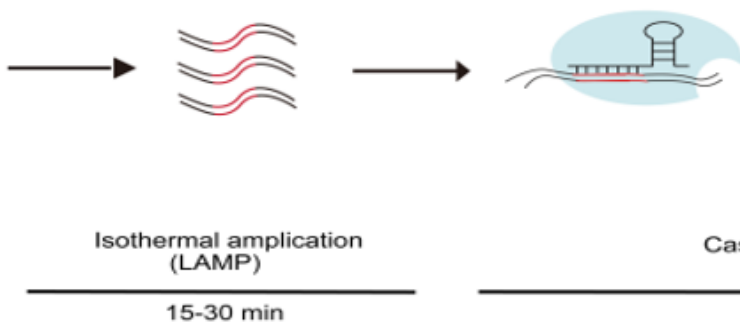

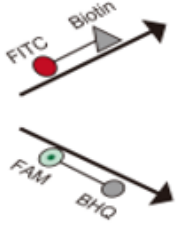

Cas12a-DETECTR

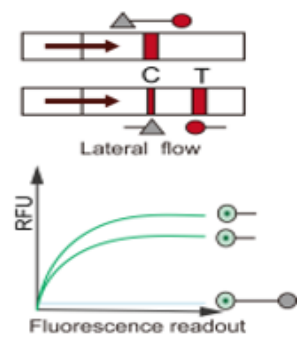

$15-30 \mathrm{~min}$

Figure 1. Cas12a-DETECTR assay of HBV DNA. After HBV serum samples were rapidly processed within 10 min, the extracted HBV DNA was amplified by performing LAMP of the target sequence (15-30 min). Cognate binding of the Cas12a-crRNA complex to amplified HBV DNA targets triggered the collateral activity of Cas12a, which cleaved ssDNA reporters (15-30 $\mathrm{min})$. The cleaved ssDNA is visualized by both fluorescence readout (FAM-BHQ) and lateral flow test strips (FITC-Biotin).

\section{Results}

\subsection{Construction of Cas12a-DETECTR System for HBV Detection}

For Cas12a-DETECTR to detect various HBV subtypes, all HBV polymerase coding region 9199 sequences were sequence aligned to screen out conserved target regions, and then two crRNAs were designed accordingly (Figure 2a, Table S5). After the Cas12a assay with $10^{7}$ copies / $\mu \mathrm{L}$ standard plasmid, the results showed that crRNA1 was efficient. Therefore, crRNA1 was selected for the subsequent detection of HBV in Cas12a-DETECTR (Figure 2c). PrimerExplorer v5 software was used to design three LAMP primers. After amplification of the $10^{7}$ copies/ $\mu \mathrm{L}$ of standard plasmid, followed by agarose gel elec- 
trophoresis experiments, the results showed that primer 3 amplification efficiency was the best for the subsequent LAMP-Cas12a assay (Figure 2b,d, Table S5).

a

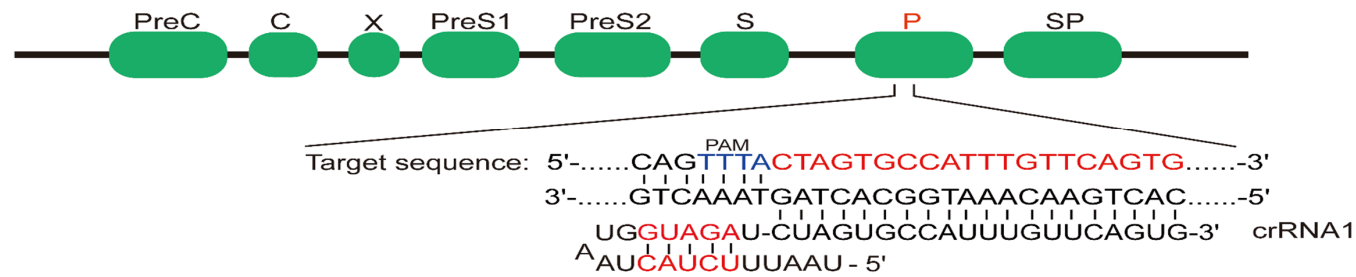

$\mathrm{F} 2$

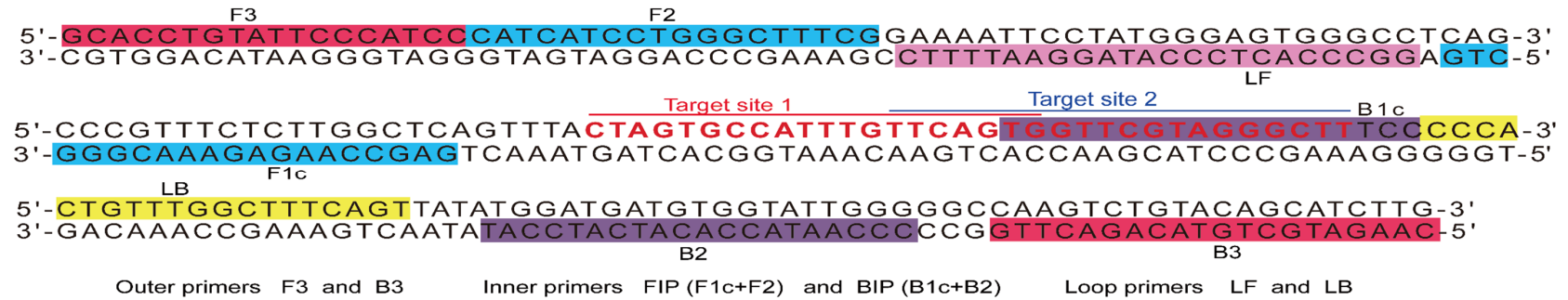

C

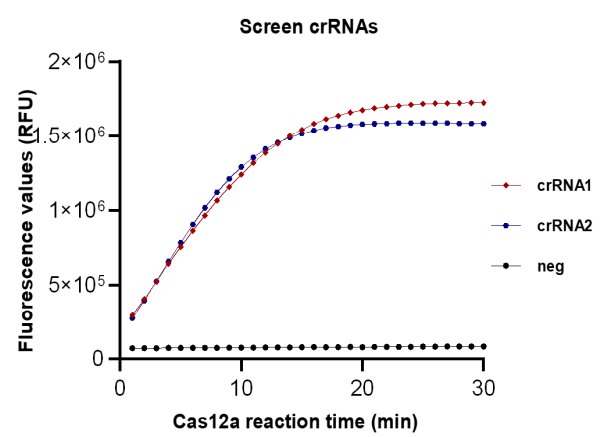

d

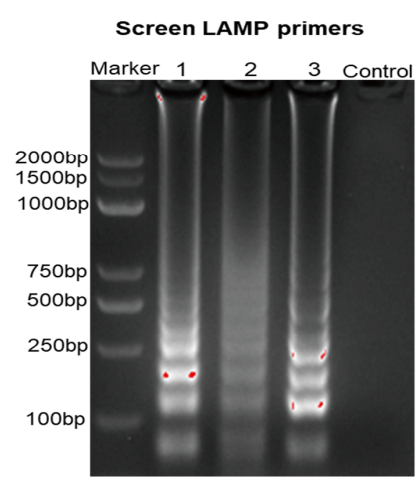

Figure 2. Preparation of Cas12a-DETECTR system. (a) HBV genome map shows target and crRNA sequences on the polymerase coding region. (b) The sequence and location of LAMP primers and target sites in the HBV genome. (c) To screen good crRNA, we performed Cas12a assays on $10^{7}$ copies/ $\mu \mathrm{L}$ of HBV plasmids by using crRNA 1 and 2; neg: parallel control without crRNA addition for the same Cas12a assay. (d) To screen the best LAMP primer, after the amplification of different LAMP primers, we performed agarose gel electrophoresis.

\subsection{Analysis of the LoD and Specificity of the Cas12a-DETECTR System}

The limit of detection (LoD), specificity, and detection time were used to evaluate the newly established HBV-DETECTR assay. A series of gradient dilutions of HBV standard plasmids was amplified using LAMP for $30 \mathrm{~min}$ and detected using the Cas12a-DETECTR fluorescence readout. The Cas12a-DETECTR assay had a low LoD of 1 copy $/ \mu \mathrm{L}$, while the variation from the negative control could be distinguished in 2 min (Figure 3a,b).

Because the LOD results showed that the Cas12a-DETECTR assay was particularly sensitive, we therefore further explored the LOD of the Cas12a-DETECTR assay in terms of time. The HBV plasmids of 1 copy $/ \mu \mathrm{L}$ were amplified by LAMP for different times, and then the Cas12a-DETECTR assay was performed. The results showed that after $3 \mathrm{~min}$ of Cas12a reaction, a significant difference was observed in the fluorescence values between the LAMP amplification for $10 \mathrm{~min}$ and the negative control. Hence, 1 copy/ $\mu \mathrm{L}$ of $\mathrm{HBV}$ DNA could be detected by LAMP-Cas12a fluorescence readout within $13 \mathrm{~min}$ (Figure 3c,d).

In addition, the fluorescence readout results of the Cas12a-DETECTR assay showed that the fluorescence curve of LAMP amplification at $15 \mathrm{~min}$ and afterward was consistent (Figure 3c,d). To ensure the stability of the test results, we performed LAMP amplification for $15 \mathrm{~min}$ as the subsequent Cas12a lateral flow test strip incubation time gradient test. 
The results of the lateral flow test strips showed that the Cas12a-DETECTR showed positive results after $5 \mathrm{~min}$ of incubation. Next, after $15 \mathrm{~min}$ of incubation, all reporter molecules in the Cas12a detection system were cleaved, showing strong positive results (Figure 3e).

a

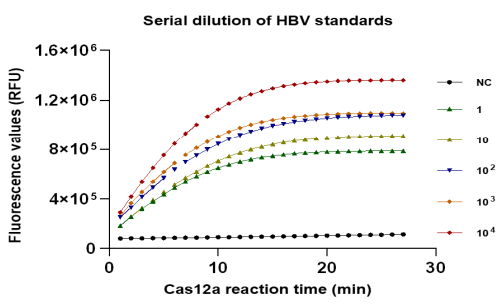

C

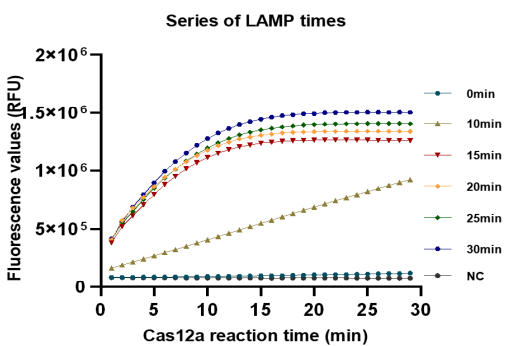

Cas12a-DETECTR specificity

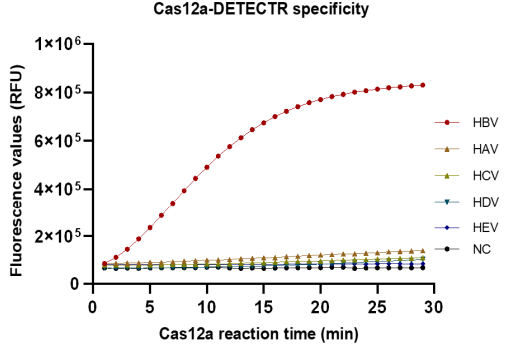

b

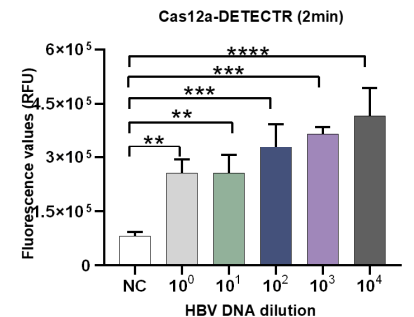

Cas12a-DETEC TR detection (3min)

d

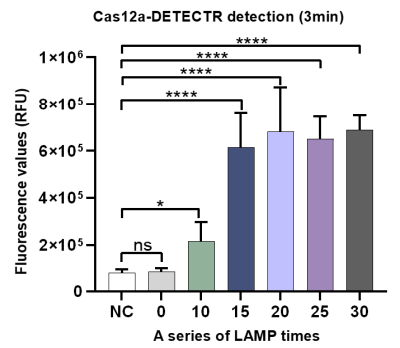

g

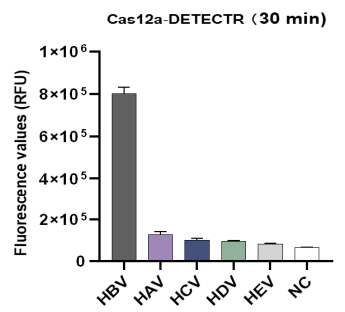

e

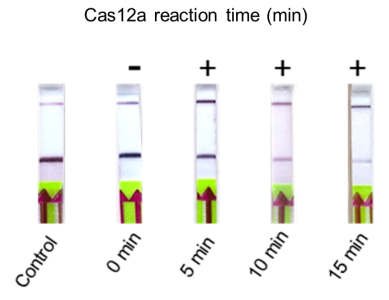

$\mathrm{h}$

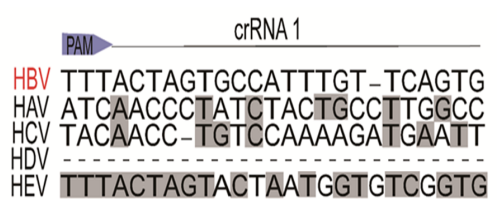

Figure 3. Determination of LoD and specificity of Cas12a-DETECTR assay. (a) Fluorescence curves generated by the Cas12a-DETECTR reaction at each dilution. Data were expressed as mean \pm SD. from triplicate assays. Negative control (NC) utilized RNase-free water as input instead of HBV DNA dilutions. (b) Comparison of fluorescence values generated after $2 \mathrm{~min}$ of Cas12a-DETECTR reaction at each dilution. (c) Fluorescence curves generated by the Cas12a-DETECTR reaction at each LAMP incubation time gradient (HBV concentration is 1 copy $/ \mu \mathrm{L}$ ). (d) Comparison of fluorescence values generated after $3 \mathrm{~min}$ of Cas12a-DETECTR reaction at each LAMP incubation time gradient (HBV concentration, 1 copy $/ \mu \mathrm{L}$ ). (e) Comparison of lateral flow test strip results at each Cas12a-DETECTR reaction time gradient after $15 \mathrm{~min}$ of LAMP incubation (HBV concentration is 1 copy/ $\mu \mathrm{L}$ ). (f) HBV Cas12a-DETECTR fluorescence readout assays for different hepatitis virus clinical samples. (g) The fluorescence values generated after $30 \mathrm{~min}$ of Cas12a-DETECTR reaction for each virus. (h) The sequence alignment of nucleotides in the crRNA 1 target region of the HBV gene with other common human hepatitis viruses, including $\mathrm{HAV}, \mathrm{HCV}, \mathrm{HDV}$, and HEV. + : indicates a positive sample, $-:$ indicates a negative sample. ${ }^{* *}: p<0.01$; ***: $p<0.001 ; * * * *: p<0.0001$.

Importantly, the specificity of the newly established HBV-Cas12a assay platform was tested. First, we performed a BLAST analysis of the target sequences in crRNA1 and found no similar sequences except for each subtype of HBV. However, the sequence alignment with other hepatitis viruses (including HAV, HCV, HDV and HEV) revealed significant differences (Figure 3h). Next, we used Cas12a-DETECTR to perform identification assays for various hepatitis viruses. The assay results showed that Cas12a-DETECTR could specifically identify only HBV (Figure 3f,g). 


\subsection{Validation of DETECTR-Based HBV Assay for Clinical Samples}

To assess the effectiveness of the HBV-DETECTR assay system in clinical samples, 73 serum samples were assayed using qPCR and Cas12a-DETECTR (Table S7). The serum samples for the Cas12a-DETECTR assay were used for pre-processing using ultra rapid nucleic acid releaser. To improve the stability and sensitivity of the Cas12a assay platform in clinical validation, we adjusted and optimized the Cas12a-DETECTR system (Table S4). Clinical samples were assayed using the adjusted Cas12a-DETECTR assay system by using fluorescent readout and lateral flow strip techniques. The results show that HBV detection based on the Cas12a-DETECTR's fluorescence readout and lateral flow test strip method was consistent with the qPCR assay (Figure 4). The sensitivity, specificity, PPA, NPA, Youden's index, and the ROC curve results showed an area under the ROC curve of 1, indicating that DETECTR-based HBV detection was fully comparable to qPCR (Table 1, Figure S1). Moreover, the Cas12a-DETECTR assay has advantages in terms of sample processing, assay time, and assay difficulty, and is fully capable of point-of-care testing of HBV samples (Table 2).

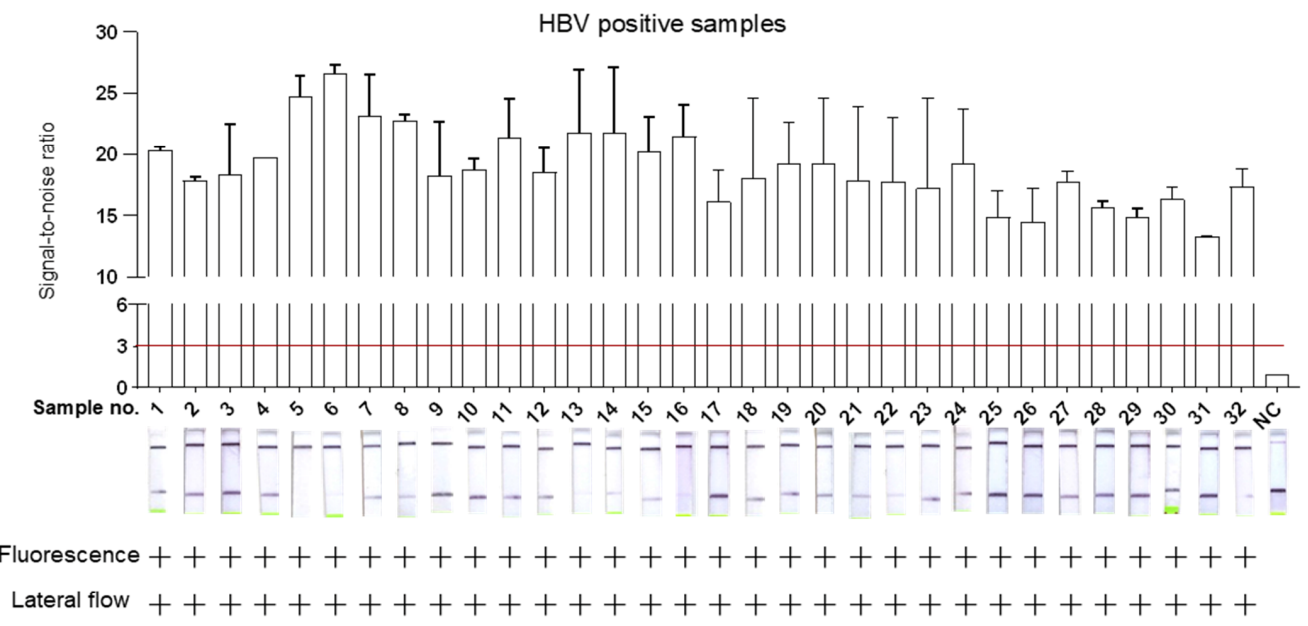

HBV negative samples

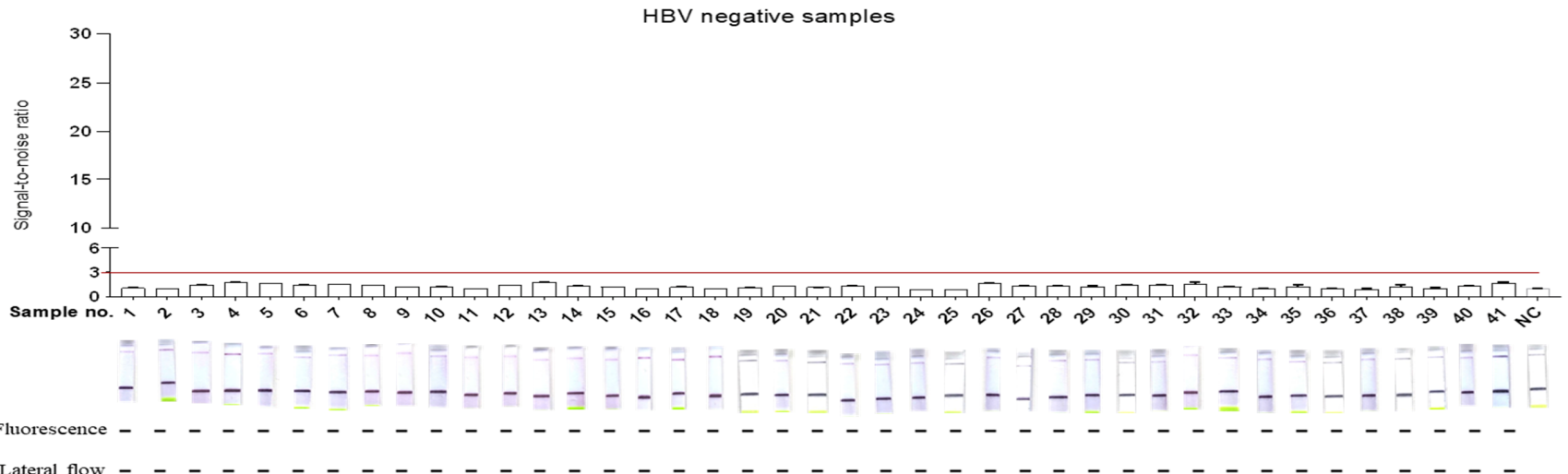

Figure 4. Cas12a-DETECTR assay for HBV in 73 clinical samples, which were tested using qPCR and Cas12a-DETECTR (including fluorescence readout and lateral flow test strip readout). Evaluation of Cas12a-DETECTR assay results with qPCR assay results. HBV positivity was defined based on $\mathrm{QPCR}$ results. +: indicates a positive sample, -: indicates a negative sample. 
Table 1. Clinical validation of a DETECTR-based assay for the detection of HBV.

\begin{tabular}{|c|c|c|c|c|c|c|c|c|}
\hline & & \multicolumn{2}{|c|}{ qPCR } & \multirow{2}{*}{$\begin{array}{c}\text { Sensitivity } \\
(95 \% \mathrm{CI})\end{array}$} & \multirow{2}{*}{$\begin{array}{c}\text { Specificity } \\
(95 \% \mathrm{CI})\end{array}$} & \multirow{2}{*}{$\begin{array}{c}\text { PPA } \\
(95 \% \mathrm{CI})\end{array}$} & \multirow{2}{*}{$\begin{array}{c}\text { NPA } \\
(95 \% \text { CI })\end{array}$} & \multirow{2}{*}{$\begin{array}{c}\mathrm{YI} \\
(95 \% \mathrm{CI})\end{array}$} \\
\hline & & Positive & Negative & & & & & \\
\hline \multirow{3}{*}{$\begin{array}{l}\text { Cas12a-DETECTR } \\
\text { fluorescence readout }\end{array}$} & Positive & 32 & 0 & $100 \%$ & $100 \%$ & $100 \%$ & $100 \%$ & $100 \%$ \\
\hline & Negative & 0 & 41 & \multirow{2}{*}{$(86.7-100 \%)$} & \multirow{2}{*}{$(89.3-100 \%)$} & \multirow{2}{*}{$(86.7-100 \%)$} & \multirow{2}{*}{$(89.3-100 \%)$} & \multirow{2}{*}{$(76-100 \%)$} \\
\hline & Total & 32 & 41 & & & & & \\
\hline \multirow{3}{*}{$\begin{array}{l}\text { Cas12a-DETECTR } \\
\text { lateral-flow readout }\end{array}$} & Positive & 32 & 0 & $100 \%$ & $100 \%$ & $100 \%$ & $100 \%$ & $100 \%$ \\
\hline & Negative & 0 & 41 & \multirow{2}{*}{$(86.7-100 \%)$} & \multirow{2}{*}{$(89.3-100 \%)$} & \multirow{2}{*}{$(86.7-100 \%)$} & \multirow{2}{*}{$(89.3-100 \%)$} & \multirow{2}{*}{$(76-100 \%)$} \\
\hline & Total & 32 & 41 & & & & & \\
\hline
\end{tabular}

73 samples were used for clinical validation of the DETECTR assay. CI, confidence interval; PPA, positive predictive agreement; NPA, negative predictive agreement; YI, Youden's index; the gold standard for HBV is qPCR.

Table 2. Comparison of Ca12a-DETECTR assay with existing assays.

\begin{tabular}{|c|c|c|c|c|}
\hline & $\mathrm{qPCR}^{\mathrm{a}}$ & ELISA $^{b}$ & PCR-Cas13a & LAMP-Cas12a \\
\hline Assay type & Nucleic acid assay & $\begin{array}{l}\text { Antigen and antibody } \\
\text { assay }\end{array}$ & Nucleic acid assay & Nucleic acid assay \\
\hline LoD & $30 \mathrm{IU} / \mathrm{mL}$ & Poor sensitivity & 1 copy $/ \mu \mathrm{L}$ & 1 сору $/ \mu \mathrm{L}$ \\
\hline Nucleic acid extraction & Yes & No & Yes & No \\
\hline $\begin{array}{l}\text { Equipment } \\
\text { requirements }\end{array}$ & qPCR & $\begin{array}{l}\text { Enzyme-labeled } \\
\text { instrument }\end{array}$ & PCR, qPCR & Metal bath \\
\hline Complex operations & Yes & Yes & Yes & No \\
\hline Cross-reactivity & No & Yes & No & No \\
\hline Assay cost & Expensive & Economic & Economic & Economic \\
\hline $\begin{array}{l}\text { Sample-to-result time } \\
\text { (approximate) }\end{array}$ & $4 \mathrm{~h}$ & $1-2 \mathrm{~h}$ & $2-3 \mathrm{~h}$ & $60-70 \mathrm{~min}$ \\
\hline point-of-care testing & No & No & No & Yes \\
\hline Clinical Application & $\begin{array}{c}\text { Quantitative HBV } \\
\text { DNA assay }\end{array}$ & $\begin{array}{c}\text { Assisted qualitative } \\
\text { assay }\end{array}$ & $\begin{array}{l}\text { Pending clinical } \\
\text { validation }\end{array}$ & $\begin{array}{l}\text { Pending clinical } \\
\text { validation }\end{array}$ \\
\hline
\end{tabular}

a Sansure Biotech's Hepatitis B Virus Nucleic Acid Assay Kit (Prominence HBV DNA) for qPCR Detection Kit; ${ }^{\mathrm{b}}$ HBV serological test refer to Shanghai Rongsheng Bio's Hepatitis B virus surface antibody diagnostic kit (enzyme-linked immunoassay).

\section{Discussion}

HBV infection left without timely diagnosis and treatment may be transmitted to others and may develop into chronic hepatitis B, which can cause cirrhosis and hepatocellular carcinoma [20]. In the prevention and control of hepatitis B transmission, the most suitable detection technology is still being studied [21]. Importantly, qPCR and ELISA assays may not be excellent choices for HBV testing in developing countries in the western Pacific and African regions, where the prevalence of hepatitis B is high (Table 2) [22,23]. Therefore, rapid, accurate, and economical diagnostic tests that can be deployed at point-of-care are needed to prevent and control hepatitis B transmission.

With the development of CRISPR/Cas-based systems for nucleic acid detection, rapid, accurate, and portable point-of-care detection of pathogens has become a reality. Although the highly sensitive detection of HBV based on PCR-Cas13a has been achieved, it still requires complex nucleic acid extraction of clinical samples, specialized PCR amplification, and fluorescence collection of Cas13a by the qPCR system, thus limiting the efficiency of detection and point-of-care testing [24].

Here, we developed the LAMP-Cas12a method for HBV detection to develop HBV detection. We used a rapid clinical sample processing method that allows for the extraction of nucleic acids in a short time frame, without the use of complex kits and specialized equipment, thus allowing for point-of-care testing. In the present study, we used the LAMP- 
based amplification method to eliminate the defects of previous CRISPR assays, which were its poor RPA amplification and the need for specialized equipment and time-consuming PCR amplification, while ensuring amplification efficiency [19]. More importantly, in combination with lateral flow test strip technology, the results can be visible to the naked eye, and have great practical value in home testing. Fluorescence readout can be achieved with portable fluorescence collection instruments, or with point-of-care detection by colorimetric analysis [25-27]. In the present study, the LoD of LAMP-Cas12a was 1 copy/ $\mu \mathrm{L}$. In addition, fluorescent readouts could be detected as early as $13 \mathrm{~min}$ and lateral flow test strips could be used for successful detection within 20 minutes (Figure 3a-e). More importantly, the LoD is comparable to that of qPCR, which is more advantageous in terms of efficiency and cost of detection, and does not rely on specialized equipment. However, the results were not ideal in pre-experiments of clinical sample testing. Therefore, the effect of the amount and purity of nucleic acids extracted, caused by the rapid sample pre-processing, was minimized by extending the LAMP incubation time to $30 \mathrm{~min}$. In addition, the Cas12a-DETECTR assay system was optimized (Table S4). After continuous optimization of the LAMP-Cas12a assay system and conditions, the sensitivity and specificity of the LAMP-Cas12a-based assay reached $100 \%$ for both fluorescent readouts and lateral flow test strips after validation evaluation of 73 clinical samples (Table 1, Figure 4). In addition, the method was found to be highly specific and resistant to interference (Figure $3 \mathrm{e}-\mathrm{g}$ ). Therefore, this study provides a LAMP-Cas12a-based HBV point-of-care assay that has high practical value in the future prevention and control of hepatitis $B$.

\section{Materials and Methods}

\subsection{Nucleic Acid Preparations}

All HBV polymerase coding region sequences were obtained from the HBV database (https:/ /hbvdb.lyon.inserm.fr/HBVdb/) and sequence alignment was performed using Clustal X software [28]. After sequence alignment, conserved sequences matching the Cas12a protospacer adjacent motif ( $5^{\prime}$-TTTN) were screened, and the corresponding CRISPR RNAs (crRNAs) were designed as guide RNAs (gRNAs) for subsequent HBV Cas12a assays [29,30]. Different crRNAs were performed by Cas12a-DETECTR for screening the optimal crRNA. Based on the screened optimal crRNA, the corresponding LAMP primers were designed using the PrimerExplorer v5 software (https:/ / primerexplorer. jp/e/ accessed on 29 April 2021) (Eiken Chemical Co. LTD, Tokyo, Japan). BLAST and sequence alignment were used to analyze the specificity of the designed LAMP primers and candidate target sequences for the HBV genome. Moreover, crRNAs were designed, synthesized, transcribed, and purified as previously described [16,31] (Tables S1 and S2).

\subsection{Loop-Mediated Isothermal Amplification (LAMP)}

LAMP primers were synthesized by Shanghai Sangon Biotech. The LAMP reaction system was operated strictly according to the instructions (Sangon Biotech, Shanghai, China), and the amplification was carried out at $65^{\circ} \mathrm{C}$ for $15-30 \mathrm{~min}$ (Table S3). In addition, the amplified LAMP product was centrifuged and then placed on ice to prevent residual aerosol contamination on the EP tube lid.

\subsection{HBV DNA Detection Based on Cas12a-DETECTR}

The DETECTR assay was performed via steps. First, HBV DNA was pre-amplified using LAMP to generate more target sequence substrates for the Cas12a-DETECTR. Next, the detection of the amplified HBV DNA by Cas12a-DETECTR triggered the collateral cleavage of reporter molecules for lateral-flow or fluorescence assays, and this process was performed as previously described (Table S4) [15]. For Cas12a-DETECTR fluorescence readout, the $\mathrm{ABI}$ fast 7500 was used to collect in real-time the fluorescence generated by the Cas12a cleavage of the fluorescent reporter $\left(5^{\prime}-/ 6-\mathrm{FAM} / \mathrm{TTTTTT} / \mathrm{BHO} /-3^{\prime}\right)$. The lateral flow test strip method enables visual field detection and is based on the principle that after Cas12a is activated to cleave the reporter ( $5^{\prime}-/ 6$-FITC/TTTTTTT/Biotin/- $\left.3^{\prime}\right)$, the biotin end 
is captured in the first line (control line) and 6-FITC produces a positive band visible to the naked eye in the second line (test line) after binding to the anti-FITC and gold particles. In addition, the lateral flow test strip method requires incubation in a metal bath at $37^{\circ} \mathrm{C}$ for 15-30 min before performing the test [32] (Figure 1).

\subsection{Clinical Sample Collection and DNA Extraction}

Clinical serum samples were provided by the First Affiliated Hospital of Zhengzhou University. Serum samples were collected by drawing $2 \mathrm{~mL}$ of venous blood from the subject with a sterile syringe, which was then injected into a sterile collection tube, followed by direct centrifugation at $1600 \mathrm{rpm}$ for $5 \mathrm{~min}$ at room temperature to separate the serum. Finally, the sample was transferred into a $1.5 \mathrm{~mL}$ sterilized centrifuge tube for backup. Rapid HBV nucleic acid extraction was performed using ultra-rapid nucleic acid releaser (Table S6). According to the instructions, $5 \mu \mathrm{L}$ of nucleic acid releaser was added into a $1.5 \mathrm{~mL}$ centrifuge tube. Then, $20 \mu \mathrm{L}$ of HBV serum was added, and the solution was mixed gently. Then, the sample was placed in a constant-temperature metal bath, at $95^{\circ} \mathrm{C}$, for $5 \mathrm{~min}$. Finally, after equilibration at room temperature for $2 \mathrm{~min}$, the supernatant was centrifuged at 10,000 rpm for $2 \mathrm{~min}$. Exactly $5 \mu \mathrm{L}$ of supernatant was aspirated for LAMP amplification. To avoid the influence of subjective factors, we subjected the clinical samples in a blinded test by using the Cas12a-DETECTR assay (both the fluorescent readout and the lateral flow test strip method) and qPCR, respectively. Clinical samples were evaluated using qPCR as the gold standard, which is commonly used in clinical practice. In addition, the sensitivity, specificity, positive predictive value (PPV), negative predictive value (NPV), Youden's index and receiver operating characteristic curve (ROC) were used to evaluate the effectiveness of the Cas12a-DETECTR assay. For the definition of positive samples for fluorescence readout, we set the signal-to-noise ratio parameter (the ratio of the fluorescence value of the sample to the negative control, $\mathrm{S} / \mathrm{N}$ ) to $\mathrm{S} / \mathrm{N}>3$ after $30 \mathrm{~min}$ of Cas12a reaction, which was considered an HBV-positive sample.

\subsection{Statistical Analysis}

The Cas12a-DETECTR assay was performed in three parallel experiments to avoid experimental error. Data were analyzed and graphed using SPSS 21 and GraphPad Prism 8.3.0 (GraphPad, Inc., La Jolla, CA, USA).

Supplementary Materials: The following are available online at https:/ / www.mdpi.com/article/ 10.3390/ijms22094842/s1, Figure S1: ROC curve of Cas12a-DETECTR assay, Table S1: First add to the PCR tube according to the system, Table S2: The above products were mixed in the following system, Table S3: LAMP reaction systems, Table S4: Reaction system of Cas12a-DETECTR, Table S5: Sequences involved in this study, Table S6: Information on purchased reagents, Table S7: Results of qPCR for 73 clinical serum samples.

Author Contributions: R.D., S.C. and G.D. conceived and designed the experiments; R.D. performed the experiments; J.L. and M.Y. analyzed the data; Y.J., H.Y., H.L., X.Z. and Y.S. contributed reagents/materials/analysis tools; H.Y. wrote the paper. All authors have read and agreed to the published version of the manuscript.

Funding: The work was supported by the National Science and Technology Specific Projects (2018ZX10301407), and The Key Scientific Research Projects in Colleges and Universities of Henan Province (20A330004). National Natural Science Foundation of China (NO.82073618 and NO.82002147).

Conflicts of Interest: The authors declare no conflict of interest.

\section{References}

1. Liaw, Y.-F. Antiviral therapy of chronic hepatitis B: Opportunities and challenges in Asia. J. Hepatol. 2009, 51, 403-410. [CrossRef] [PubMed]

2. Gonzãlez, R.; Torres, P.; Castro, E.; Barbolla, L.; Candotti, D.; Koppelman, M.; Zaaijer, H.L.; Lelie, N.; Allain, J.-P.; Echevarrãa, J.-M. Efficacy of hepatitis B virus (HBV) DNA screening and characterization of acute and occult HBV infections among blood donors from Madrid, Spain. Transfusion 2009, 50, 221-230. [CrossRef] 
3. Schweitzer, A.; Horn, J.; Mikolajczyk, R.T.; Krause, G.; Ott, J.J. Estimations of worldwide prev-alence of chronic hepatitis B virus 279 infection: A systematic review of data published between 1965 and 2013. Lancet 2015, 386, 1546-1555. [CrossRef]

4. Tan, M.; Bhadoria, A.S.; Cui, F.; Tan, A.; Van Holten, J.; Easterbrook, P.; Ford, N.; Han, Q.; Lu, Y.; Bulterys, M.; et al. Estimating the proportion of people with chronic hepatitis B virus infection eligible for hepatitis B antiviral treatment worldwide: A systematic review and meta-analysis. Lancet Gastroenterol. Hepatol. 2021, 6, 106-119. [CrossRef]

5. Hellard, M.E.; Chou, R.; Easterbrook, P. WHO guidelines on testing for hepatitis B and C-meeting targets for testing. BMC Infect. Dis. 2017, 17, 703. [CrossRef]

6. Clements, C.J.; Baoping, Y.; Crouch, A.; Hipgrave, D.; Mansoor, O.; Nelson, C.B.; Treleaven, S.; van Konkelenberg, R.; Wiersma, S. Progress in the control of hepatitis B infection in the Western Pacific Region. Vaccine 2006, 24, 1975-1982. [CrossRef]

7. Rybicka, M.; Bielawski, K.P. Recent Advances in Understanding, Diagnosing, and Treating Hepatitis B Virus Infection. Microorganisms 2020, 8, 1416. [CrossRef]

8. Datta, S.; Chatterjee, S.; Veer, V. Recent advances in molecular diagnostics of hepatitis B virus. World J. Gastroenterol. 2014, 20, 14615-14625. [CrossRef]

9. Costa, C.I.; Delgado, I.F.; da Costa, J.A.C.; de Carvalho, R.F.; Júnior, S.D.S.M.E.; Vianna, C.O.A.; de Moraes, M.T.B. Establishment and validation of an ELISA for the quantitation of HBsAg in recombinant hepatitis B vaccines. J. Virol. Methods 2011, $172,32-37$. [CrossRef]

10. Pfefferkorn, M.; Böhm, S.; Schott, T.; Deichsel, D.; Bremer, C.M.; Schröder, K.; Gerlich, W.H.; Glebe, D.; Berg, T.; Van Bömmel, F. Quantification of large and middle proteins of hepatitis B virus surface antigen (HBsAg) as a novel tool for the identification of inactive HBV carriers. Gut 2018, 67, 2045-2053. [CrossRef]

11. Liu, W.; Liu, L.; Kou, G.; Zheng, Y.; Ding, Y.; Ni, W.; Wang, Q.; Tan, L.; Wu, W.; Tang, S.; et al. Evaluation of Nucleocapsid and Spike Protein-Based Enzyme-Linked Immunosorbent Assays for Detecting Antibodies against SARS-CoV-2. J. Clin. Microbiol. 2020, 58, 00461-20. [CrossRef] [PubMed]

12. Knott, G.J.; Doudna, J.A. CRISPR-Cas guides the future of genetic engineering. Science 2018, 361, 866-869. [CrossRef] [PubMed]

13. Myhrvold, C.; Freije, C.A.; Gootenberg, J.S.; Abudayyeh, O.O.; Metsky, H.C.; Durbin, A.F.; Kellner, M.J.; Tan, A.L.; Paul, L.M.; Parham, L.A.; et al. Field-deployable viral diagnostics using CRISPR-Cas13. Science 2018, 360, 444-448. [CrossRef]

14. Patchsung, M.; Jantarug, K.; Pattama, A.; Aphicho, K.; Suraritdechachai, S.; Meesawat, P.; Sappakhaw, K.; Leelahakorn, N.; Ruenkam, T.; Wongsatit, T.; et al. Clinical validation of a Cas13-based assay for the detection of SARS-CoV-2 RNA. Nat. Biomed. Eng. 2020, 4, 1140-1149. [CrossRef] [PubMed]

15. Gootenberg, J.S.; Abudayyeh, O.O.; Lee, J.W.; Essletzbichler, P.; Dy, A.J.; Joung, J.; Verdine, V.; Donghia, N.; Daringer, N.M.; Freije, C.A.; et al. Nucleic acid detection with CRISPR-Cas13a/C2c2. Science 2017, 356, 438-442. [CrossRef]

16. Chen, J.S.; Ma, E.; Harrington, L.B.; Da Costa, M.; Tian, X.; Palefsky, J.M.; Doudna, J.A. CRISPR-Cas12a target binding unleashes indiscriminate single-stranded DNase activity. Science 2018, 360, 436-439. [CrossRef] [PubMed]

17. Harrington, L.B.; Burstein, D.; Chen, J.S.; Paez-Espino, D.; Ma, E.; Witte, I.P.; Cofsky, J.C.; Kyrpides, N.C.; Banfield, J.F.; Doudna, J.A. Programmed DNA destruction by miniature CRISPR-Cas14 enzymes. Science 2018, 362, 839-842. [CrossRef]

18. Teng, F.; Guo, L.; Cui, T.; Wang, X.-G.; Xu, K.; Gao, Q.; Zhou, Q.; Li, W. CDetection: CRISPR-Cas12b-based DNA detection with sub-attomolar sensitivity and single-base specificity. Genome Biol. 2019, 20, 132. [CrossRef]

19. Joung, J.; Ladha, A.; Saito, M.; Kim, N.-G.; Woolley, A.E.; Segel, M.; Barretto, R.P.; Ranu, A.; Macrae, R.K.; Faure, G.; et al. Detection of SARS-CoV-2 with SHERLOCK One-Pot Testing. N. Engl. J. Med. 2020, 383, 1492-1494. [CrossRef] [PubMed]

20. Seo, Y.; Yano, Y. Short- and long-term outcome of interferon therapy for chronic hepatitis B infection. World J. Gastroenterol. 2014, 20, 13284-13292. [CrossRef]

21. Yeo, Y.H.; Nguyen, M.H. Review article: Current gaps and opportunities in HBV prevention, testing and linkage to care in the United States-a call for action. Aliment Pharmacol. Ther. 2020, 53, 63-78.

22. Sciuto, E.L.; Petralia, S.; Calabrese, G.; Conoci, S. An integrated biosensor platform for extraction and detection of nucleic acids. Biotechnol. Bioeng. 2020, 117, 1554-1561. [CrossRef]

23. Akram, A.; Islam, S.M.R.; Munshi, S.U.; Tabassum, S. Detection of Hepatitis B Virus DNA among Chronic and potential Occult HBV patients in resource-limited settings by Loop-Mediated Isothermal Amplification assay. J. Viral Hepat. 2018, 25, 1306-1311. [CrossRef]

24. Wang, S.; Li, H.; Kou, Z.; Ren, F.; Jin, Y.; Yang, L.; Dong, X.; Yang, M.; Zhao, J.; Dong, N.; et al. Highly sensitive and specific detection of hepatitis B virus DNA and drug resistance mutations utilizing the PCR-based CRISPR-Cas13a system. Clin. Microbiol. Infect. 2021, 27, 443-450. [CrossRef]

25. Wang, R.; Qian, C.; Pang, Y.; Li, M.; Yang, Y.; Ma, H.; Zhao, M.; Qian, F.; Yu, H.; Liu, Z.; et al. opvCRISPR: One-pot visual RT-LAMP-CRISPR platform for SARS-cov-2 detection. Biosens. Bioelectron. 2021, 172, 112766. [CrossRef] [PubMed]

26. Zhang, W.S.; Pan, J.; Li, F.; Zhu, M.; Xu, M.; Zhu, H.; Yu, Y.; Su, G. Reverse Transcription Recombinase Polymerase Amplification 336 Coupled with CRISPR-Cas12a for Facile and Highly Sensitive Colorimetric SARS-CoV-2 Detection. Anal. Chem. 2021, 337. [CrossRef]

27. Choi, J.-H.; Lim, J.; Shin, M.; Paek, S.-H.; Choi, J.-W. CRISPR-Cas12a-Based Nucleic Acid Amplification-Free DNA Biosensor via Au Nanoparticle-Assisted Metal-Enhanced Fluorescence and Colorimetric Analysis. Nano Lett. 2021, 21, 693-699. [CrossRef]

28. Larkin, M.A.; Blackshields, G.; Brown, N.P.; Chenna, R.; Mcgettigan, P.A.; McWilliam, H.; Valentin, F.; Wallace, I.M.; Wilm, A.; Lopez, R.; et al. Clustal W and Clustal X version 2.0. Bioinformatics 2007, 23, 2947-2948. [CrossRef] [PubMed] 
29. Yamano, T.; Zetsche, B.; Ishitani, R.; Zhang, F.; Nishimasu, H.; Nureki, O. Structural Basis for the Canonical and Non-canonical PAM Recognition by CRISPR-Cpf1. Mol. Cell 2017, 67, 633-645.e3. [CrossRef] [PubMed]

30. Li, S.-Y.; Cheng, Q.-X.; Wang, J.-M.; Li, X.-Y.; Zhang, Z.-L.; Gao, S.; Cao, R.-B.; Zhao, G.-P.; Wang, J. CRISPR-Cas12a-assisted nucleic acid detection. Cell Discov. 2018, 4. [CrossRef]

31. Kellner, M.J.; Koob, J.G.; Gootenberg, J.S.; Abudayyeh, O.O.; Zhang, F. SHERLOCK: Nucleic acid detection with CRISPR nucleases. Nat. Protoc. 2019, 14, 2986-3012. [CrossRef] [PubMed]

32. Broughton, J.P.; Deng, X.; Yu, G.; Fasching, C.L.; Servellita, V.; Singh, J.; Miao, X.; Streithorst, J.A.; Granados, A.; Sotomayor Gonzalez, A.; et al. CRISPR-Cas12-based detection of SARS-CoV-2. Nat. Biotechnol. 2020, 38, 870-874. [CrossRef] [PubMed] 